\title{
Chaotic Particle Swarm Optimization for Dynamic Routing and Wavelength Assignment in All-Optical WDM Networks
}

\author{
Ali Hassan, Chris Phillips
}

\begin{abstract}
In this paper, a novel Chaotic Particle Swarm Optimization (CPSO) based scheme is proposed for solving Dynamic Routing and Wavelength Assignment problem in All-Optical WDM optical networks without any wavelength conversion. A novel fitness function is proposed to improve the connection blocking probability by taking into account normalized path length and wavelength availability over the whole path. Simulation results show that CPSO not only performs better in terms of connection blocking probability performance as compared to other heuristic schemes like Genetic Algorithms, it also presents better solution quality as compared to Particle Swarm Optimization (PSO).
\end{abstract}

Index Terms - Chaotic Particle Swarm Optimization, WDM networks, Dynamic Routing and Wavelength Assignment, Wavelength Continuity Constraint.

\section{INTRODUCTION}

$\mathrm{W}$ DM optical networking technology poses itself as an attractive candidate to meet the non-diminishing bandwidth requirements of end users, emerging applications and services. WDM networks modulate the information streams onto different carrier wavelengths, which are then multiplexed within the same optical fiber. Communication between source and destination node is done by setting up optical channels (lightpaths) between them. In all-optical wavelength routed WDM networks, the information remains in the optical domain between source and destination nodes. This removes the bottleneck of electronic speed where the transmission capacity of the optical network is limited due to optical-electrical-optical conversion required at intermediate nodes, instead of optical transmission [1, 2]. Optical cross connects $(\mathrm{OXC})$ realize the switching process in optical domain by allowing the information arriving at $\mathrm{OXC}$ to remain modulated onto a particular wavelength and simply

Manuscript received July 06, 20009. This work is supported by the EPSRC UK Research Council under Grant EP/D078741, "Machine Learning for Resource Management in Next-Generation Optical Networks".

Ali Hassan (e-mail: ali.hassan@elec.qmul.ac.uk) and Chris Phillips are with the Department of Electronic Engineering, Queen Mary University of London, E1 4NS UK. redirecting to the appropriate output, typically using MicroElectro-Mechanical Systems (MEMS).

Wavelength routed networks equipped with wavelength conversion capability at intermediate nodes are known as wavelength-interchangeable (WI) networks [3]. In such networks, a lightpath between end nodes can be set-up using different wavelengths on the links along the route. If the optical switches at intermediate nodes are not equipped with wavelength conversion capability, such networks are known as wavelength-selective (WS) networks. In this case, same wavelength needs to be employed along the entire route of a lightpath. This restriction is known as the wavelength continuity constraint [4]. Furthermore, two lightpaths sharing a common edge (i.e. link) of the network need to be assigned unique wavelengths. This constraint is known as the "wavelength clash constraint".

Connection provisioning in optical networks requires computing an appropriate route and a wavelength for the lightpath to be established. The process of route computation and assignment of wavelength is called Routing and Wavelength Assignment (RWA) process. There are two types of RWA processes: Static RWA and Dynamic RWA [5, 6]. In static RWA, all the connection requests are known in advance and the objective of RWA is to assign routes and wavelengths to all the lightpaths globally while keeping the amount of network resource required minimal [7]. Static RWA is a well known NP-Hard (Non-Polynomial Time - Hard) problem. In the case of dynamic RWA, the connection requests arrive unexpectedly with random holding time. So provisioning that connection requires assignment of route and wavelength individually. The objective of dynamic RWA is to minimize the blocking probability of the connection requests during assignment of route and wavelength. Unexpected arrival of the connection request with random stay time in the network makes dynamic RWA even more challenging. Traditional mathematical search schemes like Integer Linear Programming (ILP) and Graph Coloring become ineffective at solving the DRWA problem because of the computational complexity. Therefore, different heuristic and stochastic schemes are used to solve DRWA in a reduced amount of time. Most of the heuristic schemes used for DRWA simplify the problem domain by decomposing it into two subproblems: the routing sub-problem and a wavelength 
assignment sub-problem [7]. A detailed review of routing of wavelength assignment can be found in [7].

In this paper, a novel chaotic particle swarm optimization (CPSO) based scheme inspired by swarm intelligence is proposed to provide DRWA under the wavelength continuity constraint. The First-Fit (FF) algorithm is chosen for wavelength assignment, as it is simple to implement as compared to schemes like Max-Sum and RCL [7]. Also the performance difference among various wavelength assignment schemes is not very large [5]. The rest of the paper is organized as follows: Section II introduces the concept of Generalized Particle Swarm Optimization (PSO) and Chaotic Particle Swarm Optimization (CPSO). Section III explains the novel CPSO based scheme proposed for dynamic RWA. Section IV presents simulation results and analysis. Finally conclusions are presented in Section V.

\section{ChaOtic PARTicle SWARM OPTIMIZATION (CPSO)}

\section{A. Generalized Particle Swarm Optimization (PSO)}

PSO is a population based evolutionary optimization algorithm, originally developed by Kennedy and Eberhart [8], inspired by social behaviour of bird flocking (and schools of fish). In PSO, a swarm is a collection of population members called 'particles' which evolves over time for problem solving. Each particle in the swarm has both a position and velocity. The position of the particle in multidimensional problem space represents a candidate solution. The velocity of the particles moves it from one position to another position over the problem search space. In order to avoid the velocity from becoming very large in the initial PSO iteration, and to avoid premature convergence to a local optima, a number of improvements are suggested in the literature [9, 10, and 11]. For example, Clerc [12] proposed the use of constriction factor ' $\chi$ ' in order to prevent large velocity values. The PSO equation where the position and velocity represents physical attributes of the particles is represented by (1) and (3).

Calculating a Single Particle's New Velocity

$V_{i d}=\chi\left[V_{i d}+\eta_{1} r_{1}\left(P_{i d}-X_{i d}\right)+\eta_{2} r_{2}\left(P_{i d}^{a}-X_{i d}\right)\right] i=12, \ldots, N d=1,2, . D$.

Where,

$\chi=2\left(\left|2-\eta-\sqrt{\eta^{2}-4 \eta}\right|\right)^{-1}$ if $\eta=\eta_{1}+\eta_{2}>4$

"Moving" a Single Particle in a Swarm

$X_{i d}=X_{i d}+V_{i d}$

In Equation 1 and 3,

$P_{i d}$ is the personal best position, a particle has reached;

$\mathrm{P}_{\mathrm{id}}{ }^{\mathrm{n}}$ is the global best position of all the particles.

' $\eta_{1}$ ' (the self-confidence factor) and ' $\eta_{2}$ ' (the swarmconfidence factor) are positive constants called 'acceleration constants' to determine the influence of $P_{i d}$ and $P_{i d}{ }^{n}$.

' $r_{1}$ ' and ' $r_{2}$ ' are independent random numbers in the range $[0,1]$.
' $\mathrm{N}$ ' is the total number of particles in the swarm.

' $D$ ' is the dimension of the problem search space i.e. the number of function parameters being optimized.

PSO starts by randomly initializing the position and velocities of all the particles in the swarm over the problem space. The position of $i^{\text {th }}$ particle is represented by the vector $\mathrm{X}_{\mathrm{i}}=\left[\mathrm{X}_{\mathrm{i} 1}, \mathrm{X}_{\mathrm{i} 2} \ldots \mathrm{X}_{\mathrm{iD}}\right]$ and velocity of $\mathrm{i}^{\text {th }}$ particle is represented by the vector $V_{i}=\left[V_{i 1}, V_{i 2} \ldots V_{i D}\right]$. For each iteration (until the convergence criteria is met), the fitness function is applied to the particles to quantize their respective positions over the problem search space. The particle with the best fitness value in the neighborhood is marked as the global/local best particle. Each particle will also keep a record of its personal best position searched so far. Equation (1) is used to calculate new velocity for each particle in the swarm based on particle's previous velocity, its current and personal best position, and the position of the particle with best fitness value in the neighborhood. Equation (3) is then used to apply the velocity to the particle. As a result of this, the particle will move to a new position i.e. it will now represent a new candidate solution to the problem being studied.

\section{B. Chaotic Particle Swarm Optimization (CPSO)}

One problem with PSO is the problem of being trapped in local optima which can lead to premature convergence of the swarm members. Recently, many studies have been presented to improve the performance of PSO based on chaotic search behaviour. To improve the performance of PSO, [13] has proposed a hybrid PSO based scheme by incorporating chaos. Simulation results of this hybrid scheme show that the chaos greatly improves the searching efficiency and searching quality of the swarm. Similarly, in [14] and [15], chaos mapping has been introduced to enhance the performance of PSO algorithms for reactive power optimization and short term hydroelectric system scheduling respectively. A chaotic PSO algorithm is proposed in [16] for image classification. The biological 'atmosphere' for the position updates of the particles is modeled by introducing wind-speed and winddirection along the flight-path of the particles, which incorporates chaotic theory in PSO. Introduction of windspeed in particle's position update, influence the motion of the swarm members by either assisting them (if the wind direction is along the flight-path) or opposing them (if the wind direction is against the flight-path) in reaching the global best particle of the swarm.

In this paper, a novel chaotic particle swarm optimization (CPSO) algorithm exploiting the concept of chaos factor in PSO [16] is proposed for dynamic routing and wavelength assignment in All-Optical WDM networks without any wavelength conversion capability. Equation (4) is used to calculate the new position of the particle instead of (3) in the case of CPSO. Three factors involved in position update are: Particle's previous position, its new velocity calculated according to (1) and the chaos factor calculated according to (5). 
$X_{i d}=X_{i d}+V_{i d}+\varphi_{w}$

where,

$\varphi_{w}=\varphi_{w}+v_{o p} * r n d_{1}+v_{f a} * r n d_{2}$

$V_{o p}=-1$ and represents the chaos factor opposing the movement of swarm particles.

$V_{f a}=+1$ and represents the chaos factor favoring the movement of the swarm particles.

$r n d_{1}$ and $r n d_{2}$ are independent random number in the range $[0,1]$.

$\varphi_{w}$ is the favorable or unfavorable chaos factor, resulting in either slowing down or speeding up the particle over the search space.

Since each particle in the swarm searches the problem space spatially separated, in different directions, so different particles will experience different conditions based on opposing or favorable chaos factor. Therefore, each particle's position is updated by the chaos-factor equation separately. In (5), when both the opposing and favorable chaos-factors are equal, it models non-chaotic PSO swarm.

\section{PROPOSED CPSO BASED SCHEME FOR DYNAMIC RWA}

\section{A. Related Work}

Like PSO, Genetic algorithms (GA) provide another evolutionary optimization approach. Genetic algorithms are swarm intelligence inspired search schemes based on the idea of natural selection and natural genetics. GA and PSO are similar in the way that both techniques are population-based search schemes that mimic the natural biological evolution and/or the social behaviour of species [17, 18]. One advantage of PSO over GA is that PSO is more computationally efficient [19]. Some performance comparison studies between GA and PSO have been reported in [17, 18, 19, 20, 21].

In [5], a novel GA based scheme is proposed to solve dynamic RWA problem in wavelength routed optical networks. In [5], a member of the population (gene) represents a route from source to destination node i.e. a candidate solution to the routing sub-problem for DRWA. Genetic operators like crossover, mutation and then selection are applied to create a new population of genes. Ammar et al. [22] has proposed a novel hybrid algorithm based on PSO and noising meta-heuristic for computing shortest paths in the network. The hybrid PSO based scheme shows better performance as compared to GA-based search algorithms for optimal shortest path computation [22]. In [6], an improved GA algorithm for solving DRWA using new fitness function is proposed to improve the performance of the genetic algorithm in [5] in terms of blocking probability. In this paper, the GA based schemes proposed in [5] and [6], are the selected schemes used for performance comparison purposes with our CPSO-based algorithm for solving the DRWA problem.

\section{B. Encoding and Decoding of Particles in the Swarm}

A typical encoding scheme for path representation is the 'direct-representation' scheme where a path is represented as a sequence of node identification numbers from the source node to the destination node. Encoding schemes based on direct representation have been used to encode paths in [23]. Gen et al. [24] proposed an indirect-representation scheme (prioritybased encoding) for solving the shortest path problem using genetic algorithms. In the priority-based encoding scheme, a path (chromosome) is represented by encoding some guiding information about a node instead of the node-ID. An example of such guiding information can be the node priority. This guiding information is used to generate a path from an arbitrary chromosome. In [25] a 'weighted encoding scheme' is used for chromosome representation in GA, whereas in [22], a cost-priority based encoding scheme is used for representing a particle in PSO.

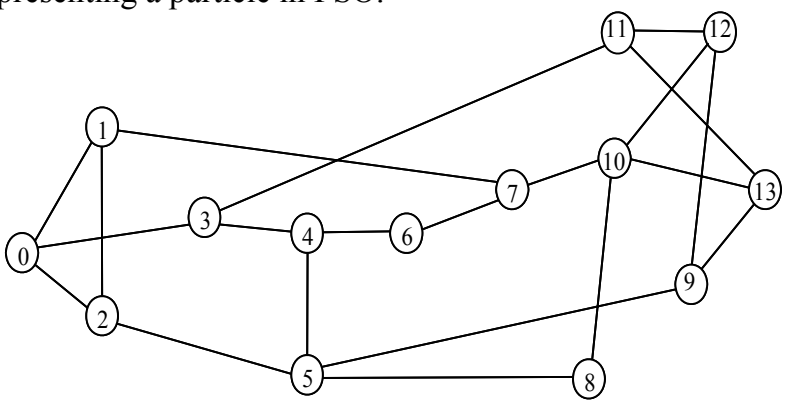

Figure 1: 14 Nodes, 21 Edges Wavelength Routing WDM Network NSFNET.

In this paper, for simplicity, a priority based encoding scheme is used. The position of the particle is represented as a vector of node priorities. The path, which a particle represents, is decoded using a path growth procedure [24] by starting from the source node and then sequentially appending the intermediate nodes one-by-one, till the destination node is reached. During the path growth procedure, if more than one node is available, the node with the highest node priority is selected. Every time as node is selected during path construction, it is marked as unavailable for the rest of path growth procedure. Figure 2 illustrates an example of priority based encoding, where a path is being constructed for a lightpath request between source node ' 1 ' and destination node ' 9 ' in NSFNET (previously shown in figure 1) by decoding the position of the particle using "path growth' procedure. 


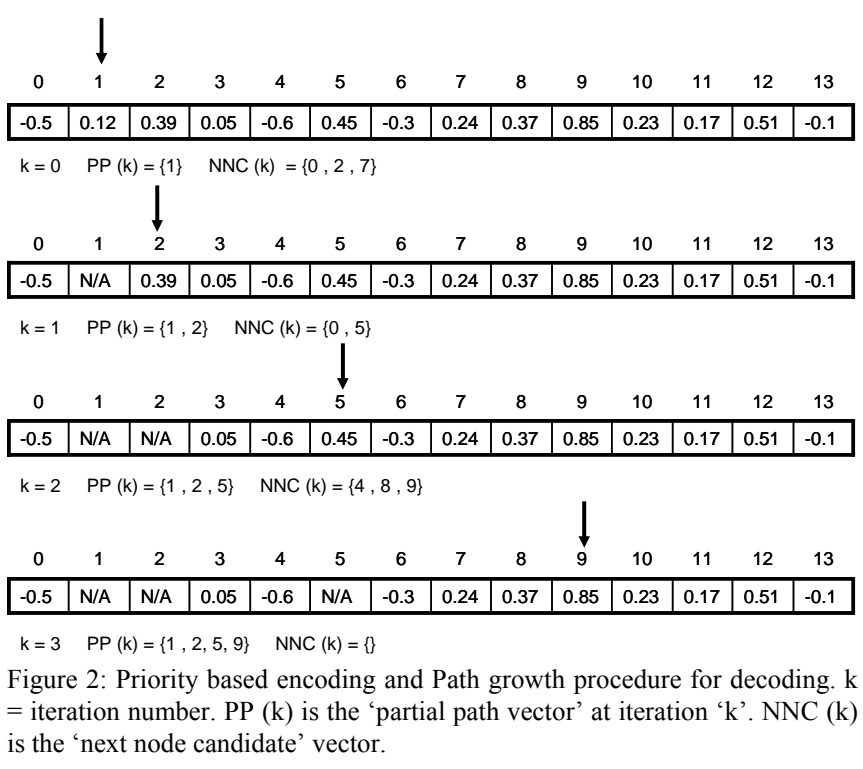

\section{Fitness Function}

In this paper, a novel fitness function is used which takes into account not only the normalized length of the route i.e. number of hops between the source and destination node. It also considers the normalized number of free wavelengths available over the whole route while satisfying wavelength continuity constraint. If no free wavelength is available for the route selected, then the fitness function will assign a large negative fitness value $(-100.0)$ for that particle.

$L_{\max }$ is the maximum length of the route between any source - destination pair and $L_{s d}$ is the length of the route between source ' $\mathrm{s}$ ' and destination ' $\mathrm{d}$ '. $\alpha[0,1]$ is a design parameter, and $W_{\text {Total }}$ is the total number of wavelengths supported by the optical network. $W_{s d}$ then defines the number of free wavelengths available over the route between source ' $\mathrm{s}$ ' and destination ' $\mathrm{d}$ '. The fitness function used in this paper is then represented by Equation (6).

$F(i)=\left[\alpha * \frac{\left(L_{\max }-L_{s d}\right)}{L_{s d}}\right]+\left[(1-\alpha) *\left(1-\frac{\left(W_{\text {Total }}-W_{\text {sd }}\right)}{W_{\text {sd }}}\right)\right]$

\section{Simulation Results AND ANALysis}

In this section, the connection blocking probability performance of the proposed Chaotic Particle Swarm Optimization based scheme (hereafter simply referred as the CPSO algorithm) for solving dynamic RWA problem is evaluated. To compare the performance of CPSO algorithm with other heuristic schemes, the Genetic Algorithms proposed in [5] and [6] are employed (hereafter simply referred as GA1 and GA2 algorithms respectively) as well as a shortest path algorithm with first-fit wavelength assignment algorithm (hereafter simply referred to as SP_FF algorithm), a simulator has been implemented in Opnet Modeler ${ }^{\mathrm{TM}}$ [26].

The main motive in choosing the two genetic based algorithms for comparison with proposed CPSO based scheme is that, $\mathrm{CPSO}$ is an evolutionary scheme belonging to the class of swarm intelligence algorithm. So it is more appropriate to produce comparisons with well-established alternative evolutionary algorithms that provide an easily understood baseline.

Experiments are conducted for NSFNET and EON networks shown in the Figure 1 and 3 respectively, where all WDM links are either assumed to have a capacity of 8 and 16 wavelengths, as indicated.

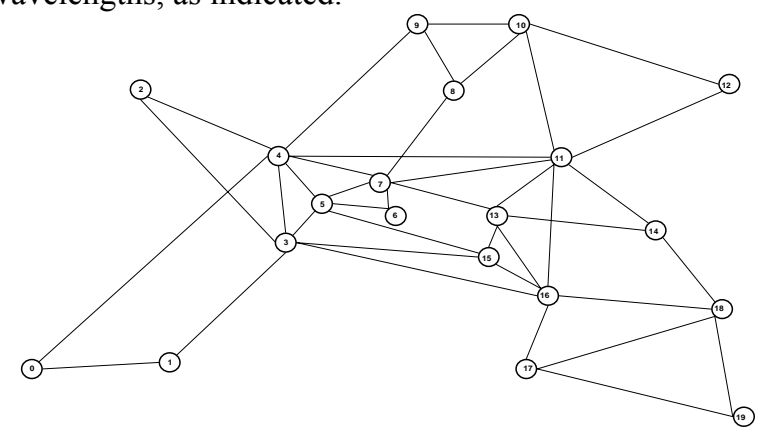

Figure 3: 20 Nodes, 39 Edges Wavelength Routing WDM Network - EON.

A performance comparison of solution quality for a simpler fitness function with varied "swarm size" and "iterations per particle" is also carried out between Particle Swarm Optimization (hereafter simply referred as PSO algorithm) and CPSO algorithm. In this paper, a global neighborhood is assumed where all the members in the swarm are particle's neighbors. During initialization, each particle's position (node priorities) and the velocity are randomly initialized with real numbers in the range $[-1.0,1.0]$. In CPSO, the initial chaosfactor is assumed to be 0.0 .

For the simulations, a dynamic traffic model is used where connection requests were generated at each node following a Poisson process with an arrival rate of $\lambda_{\text {nodes }}$. Destination nodes for the connections are randomly chosen according to a uniform distribution. Therefore, the total connection arrival rate $\left(\lambda_{\text {total }}\right)$ in the whole network is the product of total number of nodes in the network and $\lambda_{\text {nodes }}$.

If $\varphi$ is the total number nodes in the network, then

$\lambda_{\text {total }}=\varphi * \lambda_{\text {nodes }}$

The connection holding time is exponentially distributed with mean ' $\mathrm{T}$ ' seconds. Therefore, the normalized traffic load is given by Equation (8).

Normalized Traffic Load $=\lambda_{\text {total }} * T / \varphi *(\varphi-1)$

For the experiments, the value of ' $\mathrm{T}$ ' is kept constant at 50 seconds and the value of $\lambda_{\text {nodes }}$ is changed in order to vary the value of normalized traffic load. A distributed control system is used where each node in the network upon connection request arrival performs a dynamic routing and wavelength assignment computation (using out-of-band signalling). For the wavelength assignment, DIR (destination-initiated reservation) [27] along with a first-fit algorithm is used. Each node is assumed to have wavelength usage information of the whole network. No alternative routing is used and no reattempts are made for route re-computation once connection is blocked. For different traffic loads, each experiment is carried 
out 10 times with different seeds for the random number generator and the mean values are plotted in Figure 4, 5, 6 and 7 along with the $95 \%$ confidence intervals.

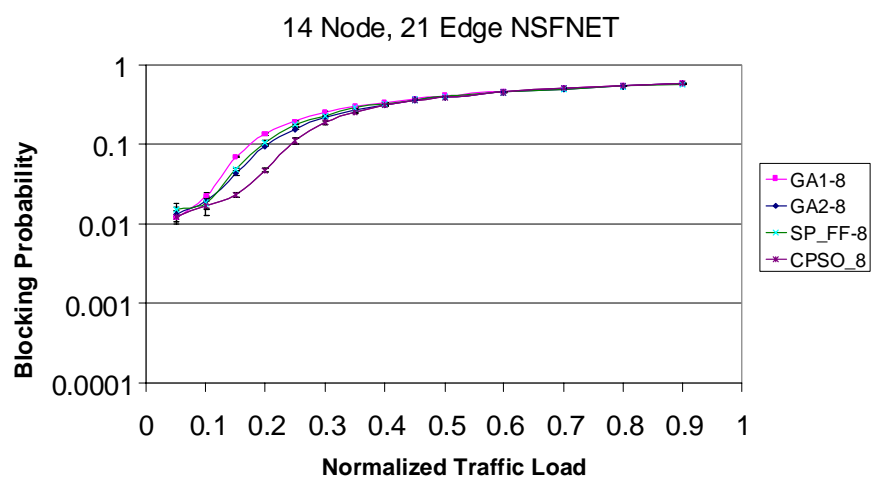

Figure 4: Blocking Probability versus Normalized Traffic load between CPSO, GA1, GA2 and SP_FF algorithms for NSFNET (Figure 1). Number of Wavelengths $=8, \alpha=0.9$, Confidence Interval $=95 \%$, Population size (for both GA and CPSO algorithms $)=15$, Iterations $(\mathrm{CPSO})=20$, Generations $(\mathrm{GA} 1$ and $\mathrm{GA} 2)=20$.

Figure 4 and 5 shows the relationship of blocking probability versus the traffic load for 8 and 16 wavelength variants of the NSFNET network respectively. As shown in Figure 4, when the wavelength capacity is 8 per link, all schemes show similar blocking probability performance at low traffic loads i.e. between normalized traffic loads of 0.05 and 0.1 . The reason is, at low traffic loads, these schemes can always find free wavelengths to be assigned whatever route they choose. When the traffic load is increased i.e. between 0.1 and 0.35 , the CPSO scheme shows a significant performance improvement in terms of blocking probability. However, when the traffic loads is increased above 0.4 , both GA and CPSO tend to the same blocking performance. The reason is, at very high traffic loads, links become saturated and the availability of free wavelengths becomes very limited as most of the wavelengths are already being used in the network. In Figure 5, the number of wavelengths supported by each link is increased to 16. At low traffic loads i.e. between 0.05 and 0.3 , all schemes show similar blocking probability performance. However, when the traffic load increases to a normalized traffic load of between 0.3 and 0.7 , CPSO performs significantly better than GA1, GA2 and the SP_FF algorithms. However, as the links saturate in terms of wavelength availability (i.e. when the normalized traffic load is above 0.7), the CPSO scheme shows comparable connection blocking probability performance to the others.

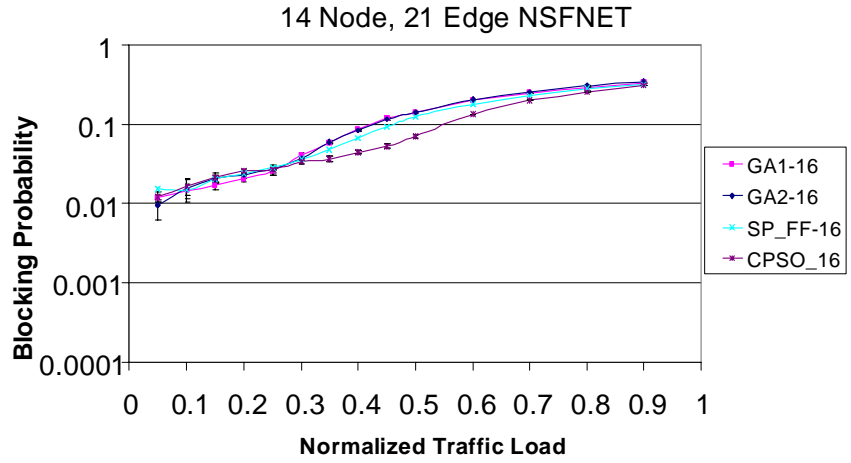

Figure 5: Blocking Probability versus Normalized Traffic load between CPSO, GA1, GA2 and SP_FF algorithms for NSFNET (Figure 1). Number of Wavelengths $=16, \alpha=0.9$, Confidence Interval $=95 \%$, Population size (for both GA and CPSO algorithms) $=15$, Iterations $(\mathrm{CPSO})=20$, Generations $(\mathrm{GA} 1$ and $\mathrm{GA} 2)=20$.

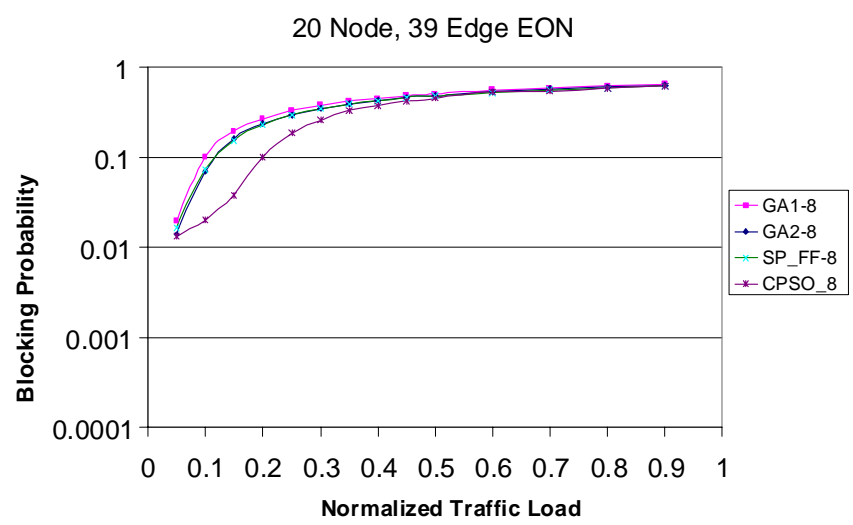

Figure 6: Blocking Probability versus Normalized Traffic load between CPSO, GA1, GA2 and SP_FF algorithms for EON (Figure 3). Number of Wavelengths $=8, \alpha=0.9$, Confidence Interval $=95 \%$, Population size (for both GA and CPSO algorithms $)=15$, Iterations $(\mathrm{CPSO})=20$, Generations $(\mathrm{GA} 1$ and $\mathrm{GA} 2)=20$.

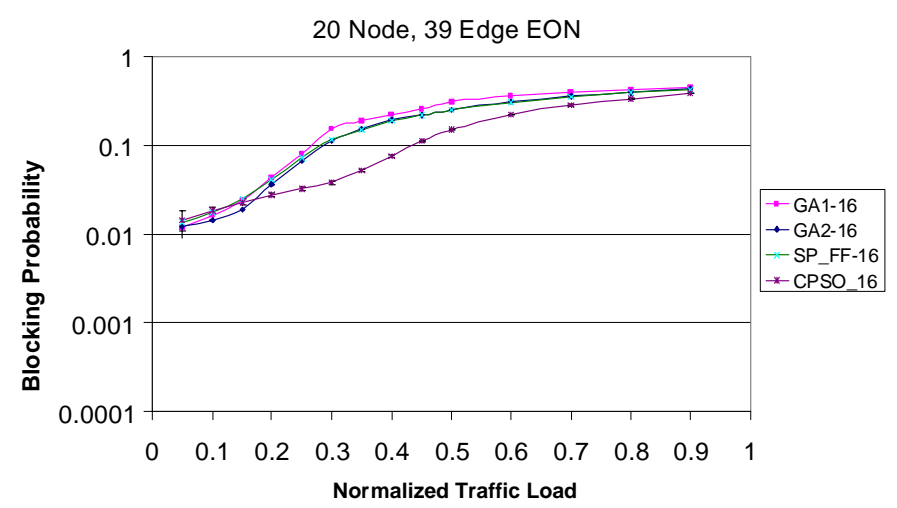

Figure 7: Blocking Probability versus Normalized Traffic load between CPSO, GA1, GA2 and SP FF algorithms for EON (Figure 3). Number of Wavelengths $=16, \alpha=0.9$, Confidence Interval $=95 \%$, Population size (for both GA and CPSO algorithms $)=15$, Iterations $(\mathrm{CPSO})=20$, Generations $(\mathrm{GA} 1$ and $\mathrm{GA} 2)=20$. 
Similar blocking probability performance behaviour can be observed in Figure 6 and 7, which show the blocking probability versus normalized traffic load for 8 and 16 wavelength based variants of EON network respectively.

In order to evaluate the performance of PSO and CPSO in terms of solution quality, an experiment is carried out where both schemes use the fitness function shown in (9) with an objective of finding the shortest route between sourcedestination node. Success ratio of an algorithm is defined as (average) number of times an algorithm finds global optimum i.e. shortest route for a connection, divided by total number of connection requests. Figure 8 shows that for a variable number of particles in the swarm, CPSO performs better than PSO in terms of success in finding shortest route. The number of iterations per particle is kept constant. Figure 9 show that even when a variable number of iterations per particles is allowed, CPSO continues to perform better than the PSO scheme. The number of particles in this case is kept constant. Figure 8 and 9 clearly show that when a varied swarm size and number of iterations are allowed, the proposed CPSO scheme presents a significantly better solution as compared to the PSO scheme.

$$
F_{s p r}=1.0 / \text { Number of hops }
$$

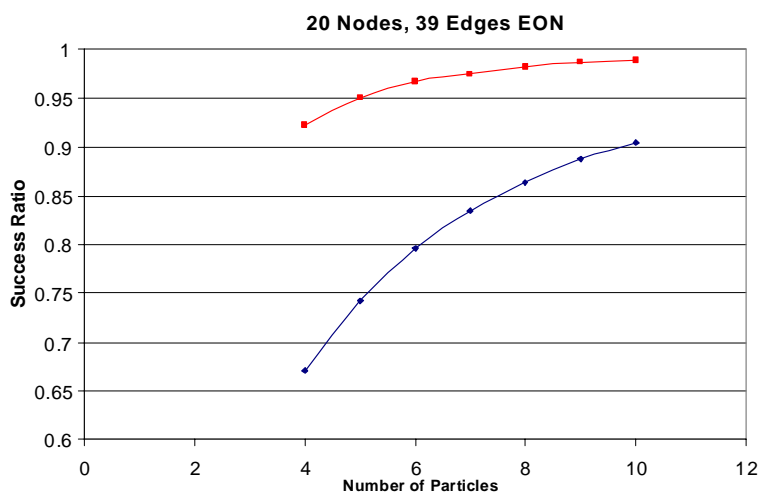

Figure 8: Success ratio in finding shortest route versus Number of particles in the swarm for PSO and CPSO in EON-network (shown in figure 3). Number of iterations allowed per particle $=10$. Normalized Network Load $=0.2$. 20 Nodes, 39 Edges EON

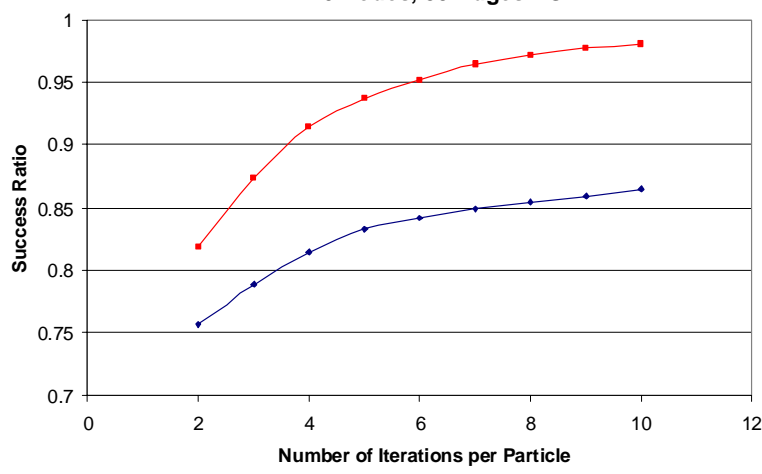

Figure 9: Success ratio in finding shortest route versus Number of iterations per particle allowed for PSO and CPSO in EON-network (shown in figure 3). Number of particles in the swarm $=8$. Normalized Network Load $=0.2$.

\section{CONCLUSIONS}

The proposed Chaotic Particle Swarm Optimization (CPSO) based scheme effectively solves dynamic RWA problem for WDM optical networks without wavelength conversion. It performs better in terms of connection blocking probability as compared to Genetic Algorithms proposed in [5], [6] and a SP FF heuristic algorithm. CPSO also provides a better quality solution as compared to PSO for a simpler fitness function with an objective of finding shortest route between source and destination node when a variable number of particles in the swarm and variable number of iterations for each particle are allowed. For these reasons, the CPSO scheme with the proposed fitness function is a suitable candidate for connection provisioning in All-Optical WDM networks.

\section{REFERENCES}

[1] Almeida. R. T. R et al. "Addressing the electronic bottleneck to virtual topology design of optical networks," Microwave and Optoelectronics Conference, 2003. IMOC 2003. Proceedings of the 2003 SBMO/IEEE MTT-S International, Publication Date: 20-23 Sept. 2003, Volume: 2, On page(s): 925- 930 vol.2, ISBN: 0-7803-7824-5, INSPEC Accession Number: 7870067.

[2] M. K. Dhodhi et al. "Bottlenecks in next generation DWDM-based optical networks," Computer Communications, Volume 24, Issue 17, 1 November 2001, Pages 1726-1733. doi:10.1016/S0140-3664(01)003577

[3] E. Karasan and E. Ayanoglu, "Effects of Wavelength Routing and Selection Algorithms on Wavelength Conversion Gain in WDM Optical Networks," IEEE/ACM Transactions on Networking, vol. 6, no. 2, pages 186-196, April 1998

[4] I. Chlamtac, A. Ganz, and G. Karmi. "Lightpath Communications: An Approach to High-Bandwidth Optical WAN's" IEEE Transactions on Communications, vol. 40, no. 7, pp. 1171-1182, July 1992.

[5] D. Bisbal et al., "Dynamic routing and wavelength assignment in optical networks by means of genetic algorithms," Photonic Network Communications, vol.7, no.1, pp.43-58, 2004.

[6] Vinh Trong Le et al. "A Genetic Algorithm for Dynamic Routing and Wavelength Assignment in WDM Networks". Publisher Springer Berlin / Heidelberg. ISSN 0302-9743 (Print) 1611-3349 (Online). Volume $3358 / 2004$. ISBN 978-3-540-24128-7. DOI 10.1007/b104574.

[7] Hui Zang et al: "A Review of Routing and Wavelength Assignment approaches for Wavelength-Routed Optical WDM networks". Optical Networks Magazine, vol. 1, no. 1(2000) 47-60.

[8] Kennedy, J. and Eberhart, R. C. (1995). "Particle swarm optimization". Proc. IEEE Int'l. Conf. on Neural Networks, IV, 1942-1948. Piscataway, NJ: IEEE Service Center.

[9] P. Srinivas and R. Battiti, "The gregarious particle swarm optimizer (GPSO)," in Proceedings of the 8th Annual Conference Genetic and Evolutionary Computation (GECCO '06), pp. 67-74, Seattle,Wash, USA, July 2006.

[10] X.-F. Xie, W.-J. Zang, and Z.-L. Yang, "Dissipative particle swarm optimization," in Proceedings of the Congress on Evolutionary Computation (CEC '02), vol. 2, pp. 1456-1461, Honolulu,Hawaii, USA, May 2002.

[11] M. Iqbal, A. A. Freitas, and C. G. Johnson, "Varying the topology and probability of reinitialization in particle swarm optimization," in Proceedings of the 7th International Conference on Artificial Evolution, Lille, France, October 2005.

[12] M. Clerc, "The swarm and queen: towards a deterministic and adaptive particle swarm optimization," in Proceedings of the Congress on Evolutionary Computation (CEC '99), vol. 3, pp. 1951-1957, Washington, DC, USA, July 1999.

[13] B. Liu, L. Wang, Y. Jin, F. Tang, D. Huang, Improved particle swarm optimization combined with chaos, Chaos Soliton. Fract. 25 (2005) 1261-1271. 
[14] C. Jiang, B. Etorre, "A hybrid method of chaotic particle swarm optimization and linear interior for reactive power optimization," Mathematics and Computers in Simulation, Volume 68, Issue 1 (February 2005), Pages: 57 - 65, ISSN:0378-4754.

[15] C. Jiang, B. Etorre, "A self-adaptive chaotic particle swarm algorithm for short term hydroelectric system scheduling in deregulated environment," Energy Conversion and Management, Volume 46, Issue 17, October 2005, Pages 2689-2696.

[16] Chandramouli, K. Izquierdo, E. "Image Classification using Chaotic Particle Swarm Optimization," Image Processing, 2006 IEEE International Conference, Publication Date: 8-11 Oct. 2006, On page(s): 3001-3004, ISBN: 1-4244-0481-9, INSPEC Accession Number: 9461981, Digital Object Identifier: 10.1109/ICIP.2006.312968

[17] E. Elbeltagi, T. Hegazy, and D. Grierson, "Comparison among five evolutionary-based optimization algorithms," Advanced Engineering Informatics, vol. 19, no. 1, pp. 43-53, 2005.

[18] R. Hassan, B. Cohanim, O. L. DeWeck, and G. Venter, "A comparison of particle swarm optimization and the genetic algorithm," in Proceedings of the 1st AIAA Multidisciplinary Design Optimization Specialist Conference, Austin, Tex, USA, April 2005.

[19] D. W. Boeringer and D. H. Werner, "Particle swarm optimization versus genetic algorithms for phased array synthesis," IEEE Transactions on Antennas and Propagation, vol. 52, no. 3, pp. 771- 779, 2004.

[20] C. R.Mouser and S. A. Dunn, "Comparing genetic algorithms and particle swarm optimization for an inverse problem exercise," The Australian \& New Zealand Industrial and Applied Mathematics Journal, vol. 46, part C, pp. C89-C101, 2005.

[21] R. C. Eberhart and Y. Shi, "Comparison between genetic algorithms and particle swarm optimization," in Proceedings of the 7th International Conference on Evolutionary Programming, pp. 611-616, Springer, San Diego, Calif, USA, March 1998.

[22] A. W. Mohemmed and N. C. Sahoo. "Efficient Computation of Shortest Paths in Networks Using Particle Swarm Optimization and Noising Metaheuristics". Hindawi Publishing Corporation, Discrete Dynamics in Nature and Society, Volume 2007, Article ID 27383, 25 pages, doi: $10.1155 / 2007 / 27383$.

[23] M.Munemoto, Y. Takai, and Y. Sato, "A migration scheme for the genetic adaptive routing algorithm," in Proceedings of IEEE International Conference on Systems, Man, and Cybernetics, vol. 3, pp. 2774-2779, San Diego, Calif, USA, October 1998.

[24] M. Gen, R. Cheng, and D. Wang, "Genetic algorithms for solving shortest path problems," in Proceedings of the IEEE International Conference on Evolutionary Computation, pp. 401-406, Indianapolis, Ind, USA, April 1997.

[25] G. Raidl and B. A. Julstrom, "A weighted coding in a genetic algorithm for the degree constrained minimum spanning tree problem," in Proceedings of the ACMSymposium on Applied Computing (SAC '00), vol. 1, pp. 440-445, Como, Italy, March 2000.

[26] Opnet Modeler, 0Hhttp://www.opnet.com

[27] Lu, K., Xiao, G. and Chlamtac, I.: Analysis of Blocking Probability for Distributed Lightpath Establishment in WDM Optical Networks. IEEE/ACM Transactions on Networking, Vol. 13, No. 1 (2005) 187197. 